\title{
TGF- $\beta 1$ is Involved in Senescence-Related Pathways in Glomerular Endothelial Cells by p16 Translocation and p21 Induction.
}

\section{Sayo Ueda}

Institute of Biomedical Sciences, Tokushima University Graduate School

\section{Tatsuya Tominaga}

Institute of Biomedical Sciences, Tokushima University Graduate School

\section{Arisa Ochi}

Institute of Biomedical Sciences, Tokushima University Graduate School

\section{Akiko Sakurai}

Institute of Biomedical Sciences, Tokushima University Graduate School

\section{Kenji Nishimura}

Institute of Biomedical Sciences, Tokushima University Graduate School

\section{Eriko Shibata}

Institute of Biomedical Sciences, Tokushima University Graduate School

\section{Shu Wakino}

Institute of Biomedical Sciences, Tokushima University Graduate School

\section{Masanori Tamaki}

Institute of Biomedical Sciences, Tokushima University Graduate School

Kojiro Nagai ( $\nabla$ kojiro.nagai@gmail.com )

Institute of Biomedical Sciences, Tokushima University Graduate School

\section{Research Article}

Keywords: cells, human, kidney, diseases, nuclear translocation, cellular senescence,

Posted Date: June 3rd, 2021

DOI: https://doi.org/10.21203/rs.3.rs-560652/v1

License: (c) (i) This work is licensed under a Creative Commons Attribution 4.0 International License. Read Full License 
Title: TGF- $\beta 1$ is involved in senescence-related pathways in glomerular endothelial cells by $\mathrm{p} 16$ translocation and p21 induction.

Authors: Sayo Ueda ${ }^{1,+}$, Tatsuya Tominaga ${ }^{1,+}$, Arisa Ochi ${ }^{1}$, Akiko Sakurai ${ }^{1}$, Kenji Nishimura ${ }^{1}$, Eriko Shibata ${ }^{1}$, Shu Wakino', Masanori Tamaki ${ }^{1}$, Kojiro Nagai $^{1, *}$

Affiliation: ${ }^{1}$ Department of Nephrology, Institute of Biomedical Sciences, Tokushima University Graduate School, Tokushima, JAPAN

${ }^{*}$ Corresponding author:

Kojiro Nagai; E-mail: kojiro.nagai@gmail.com

Tokushima University Graduate School

Address: 3-18-15, Kuramoto-cho, Tokushima, JAPAN, 770-8503

Telephone: +81-88-633-7184 Fax: +81-88-633-9245

${ }^{+}$These authors contributed equally to this work 


\section{Abstract}

p16 is an inhibitor of cyclin-dependent kinases and regulating senescence-mediated arrest as well as p21. The expression of p16 has been evaluated in human kidney diseases. However, the regulation of p16 nuclear translocation has yet to be fully investigated. TGF- $\beta 1$ is well-known to be one of the major cytokines in developing kidney diseases. TGF- $\beta 1$ can upregulate $p 21$ expression and be involved in the process of senescence. In contrast, the relationship between TGF- $\beta 1$ and $p 16$ has been poorly investigated. Here, we report the role of TGF- $\beta 1$-Smad3 pathway to regulate the $\mathrm{p} 16$ behavior in glomerular endothelial cells. To clarify the role of TGF- $\beta 1$ in the regulation of $p 16$, we analyzed podocyte-specific TGF- $\beta 1$ overexpression mice. In glomeruli, p16 was found in the nuclei of glomerular endothelial cells, leading to endothelial cellular senescence. However, the expression level of p16 was not increased in glomeruli. In cultured endothelial cells, TGF- $\beta 1$ induced nuclear translocation of p16 without the increase in p16 expression. Among human glomerular diseases, p16 was detected in the nuclei of endothelial cells. In summary, we could show 
the novel role of podocyte TGF- $\beta 1$ in the management of $\mathrm{p} 16$ behavior and cellular senescence in glomeruli, which has clinical relevance for human glomerular diseases. 


\section{Introduction}

p16 is an inhibitor of cyclin-dependent kinases. It slows down the cell cycle by prohibiting progression from G1 phase to $S$ phase. It is involved in pathways regulating senescence-mediated arrest as well as p21 ${ }^{1}$. Senescence is a cellular program that induces a stable growth arrest accompanied by distinct phenotypic alterations, including chromatin remodeling, and metabolic reprogramming ${ }^{2,3}$. A permanent arrest is effective to ensure that damaged or transformed cells do not perpetuate their genomes. This growth arrest is implemented by the activation of $\mathrm{p} 16 / \mathrm{Rb}$ and $\mathrm{p} 53 / \mathrm{p} 21$ tumor suppressor networks.

Kidney expression of p16 has been evaluated in previous reports. In human beings, a small number of p16-positive cells increasing moderately with the donors' age (up to $0.2 \%$ of all cells) was observed in the kidney. Positive p16 staining was mainly present in the tubules and was distributed in both the nucleus and cytoplasm. A few p16-positive cells were observed in the Bowman's capsule of old donors ${ }^{4,5}$. In human chronic kidney diseases, the expression of 
p16 is increased in both tubules and glomeruli in proportion to the disease progression ${ }^{6,7,8}$. However, the regulation of $\mathrm{p} 16$ nuclear translocation has yet to be fully investigated.

TGF- $\beta 1$ is well-known to be one of the major cytokines in developing kidney diseases ${ }^{9,10}$. It has been implicated in the regulation of cell proliferation, hypertrophy, apoptosis and fibrogenesis ${ }^{11}$. In particular, TGF- $\beta 1$ is considered to be the master regulator of interstitial fibrosis ${ }^{12}$. On the other hand, the evaluation of sole TGF- $\beta 1$ effect on glomerular pathological changes is limited. Hathaway et al. reported that even $200 \%$ normal levels of active TGF- $\beta 1$ protein in the plasma of non-diabetic mice could cause mesangial expansion only slightly without glomerular basement membrane thickening ${ }^{13}$, although Kopp et al. found that mice transgenic for TGF- $\beta 1$ under the control of the murine albumin promoter manifested increased hepatic expression of TGF- $\beta 1$, eight times increased plasma levels, and florid glomerulosclerosis. These findings indicated that a high level of circulating TGF- $\beta 1$ could induce increased glomerular matrix accumulation ${ }^{14}$. Ghayur et al. tried to clarify the role of the local expression of 
TGF- $\beta 1$ by inducing adenovirus-mediated gene transfer of TGF- $\beta 1$ mainly in glomerular endothelial cells. Twenty-eight days later, there were no pathological changes identified in the glomeruli in light microscopic images. However, significant proteinuria and foot process effacement was observed. The expressions of podocyte proteins such as nephrin and synaptopodin were decreased ${ }^{15}$. So far, no one has clarified the effect of the long-term locally produced TGF- $\beta 1$ on glomerular pathological changes.

Regarding senescence-related pathways, a lot of studies have emphasized the importance of TGF- $\beta 1$ signaling in the regulation of senescence both in vivo and in vitro ${ }^{16,17,18}$. TGF- $\beta 1$ can cause oxidative stress-induced activation of the p53/p21 pathway and senescence ${ }^{19}$. The Smad pathway downstream of the TGF- $\beta 1$ receptors is indeed responsible for the upregulation of $p 21$ gene $^{20,21,22}$. In contrast, there were only a few reports which suggest TGF- $\beta 1$ can increase the expression of $p 16$ even in vitro. Shimoda et al. showed TGF- $\beta 1$ induced the expression of $p 16$ in renal fibroblasts ${ }^{23}$. Kandhaya-Pillai et al. reported cell specific responses of $p 16$ expression to TGF- $\beta 1$ stimulation. 
TGF- $\beta 1$ can upregulate $p 16$ expression via activation of the mTOR pathway in preadipocytes, while TGF- $\beta 1$ can increase $p 16$ expression only with chronic interferon-y exposure in human fibroblasts ${ }^{24}$. However, to our knowledge, there is no report that shows the direct effect of TGF- $\beta 1$ on the behavior of p16 in vivo.

Therefore, in this study, we investigated the glomerular pathological changes in podocyte-specific TGF- $\beta 1$ overexpression mice. The aim of this study is to evaluate the effect of long-term locally produced TGF- $\beta 1$ overexpression in podocytes on glomerular lesion and senescence-related pathways.

\section{Results}

Characterization and pathological changes of podocyte-specific TGF- $\beta 1$ overexpression mice.

In order to evaluate the effect of long-term locally produced TGF- $\beta 1$ overexpression on glomerular lesion and senescence-related pathways, 
we mated Podocin-Cre mice with Cre-dependent TGF- $\beta 1$ overexpression mice.

Double immunostaining analysis of Podocin-Cre(+) TGF- $\beta 1$ overexpression mice (hereafter, PodCre(+) TGF mice) using the antibodies against HA tag and nephrin (podocyte marker), revealed that TGF- $\beta 1$ was expressed in podocytes (Figure 1a,b). We also confirmed the phosphorylation of Smad3 in the glomeruli of PodCre(+) TGF mice by western blot analysis (Figure 1c). Albuminuria was significantly increased (Figure 1d), while plasma TGF- $\beta 1$ concentration was not significantly changed in PodCre $(+)$ TGF mice at one year of age (mean \pm SD, Control mice: $63.8 \pm 17.7 \mathrm{ng} / \mathrm{mL}(\mathrm{N}=6)$, PodCre $(+)$ TGF mice: $77.9 \pm 42.3$ $\mathrm{ng} / \mathrm{mL}(\mathrm{N}=8))$.

Pathologically, in PodCre(+) TGF mice, collagen IV immunostained area revealed that mesangial expansion was induced significantly (Figure 1e). Mesangial expansion was confirmed by electron microscopy images (Figure 1f). Diffuse foot process effacement was prominent and glomerular basement thickening was significant (Figure 1f,g). 
Cellular senescence was induced in the glomeruli of PodCre(+) TGF mice.

In order to confirm the involvement of TGF- $\beta 1$ in senescence, we

determined the senescence associated $\beta$-galactosidase activity. $\beta$-galactosidase activity was significantly increased in the glomeruli of PodCre(+) TGF mice (Figure 2a).

The expression sites and levels of p16 and p21 in the glomeruli of PodCre(+) TGF mice.

We investigated $p 16$ and p21 expression in PodCre(+) TGF mice. The expression site was distinguished by double immunofluorescence staining with the antibody against collagen IV. Both p16 and p21 were immunostained mainly in the nuclei of endothelial cells significantly (Figure $2 b, c$ ). These findings were confirmed by the immunostaining of CD34 (endothelial cell marker) and p16 or p21 using the serial kidney sections (Supplementary Fig. S1 online).

However, in glomeruli, western blot analysis showed that the expression level of $p 16$ was not significantly changed in PodCre(+) TGF mice 
compared to that in control mice, which seemed inconsistent with the immunohistochemical analysis shown in Figure $2 b$. On the other hand, p21 expression was increased significantly in PodCre(+) TGF mice (Figure 2d).

The activation of TGF- $\beta 1$-Smad3 pathway can induce p21 expression in late phase, while it can translocate p16 to the nuclei in early phase in endothelial cells in vitro.

Next, we investigated the effect of TGF- $\beta 1$ on the expression levels of p16 and p21 in cultured endothelial cells. TGF- $\beta 1$ could increase p21 expression not in 30 minutes, but in 24 hours (late phase). SB431542, TGF- $\beta 1$ receptor antagonist, could suppress the increase in p21 expression. Transfection of constitutive active Smad3 could also increase p21 expression, suggesting p21 expression is controlled by TGF- $\beta 1$-Smad3 pathway (Figure 3a,c). However, TGF- $\beta 1$ could not affect $p 16$ expression (Figure 3a,b).

Finally, in order to clarify the inconsistency between immunohistochemical and western blot analysis in vivo, we analyzed the 
expression level of p16 in the nucleus and cytoplasm in cultured endothelial cells separately. $p 16$ was translocated to nuclei by TGF- $\beta 1$ stimulation in 30 minutes (early phase) and the effect was continued until 24 hours later. The analysis using SB431542 and constitutive active Smad3 showed the translocation was induced via TGF- $\beta 1$-Smad3 pathway (Figure 4).

\section{p16 was expressed in glomeruli of patients with kidney disease}

TGF- $\beta 1$ is involved in the development and progression of various

kidney diseases. Therefore, we evaluated the expression of p16 using human

renal biopsy samples. We found $p 16$ expression in endothelial cells in those derived from representative glomerulonephritis and nephrotic syndrome except minimal change disease and diabetic nephropathy, suggesting the common pathological significance of p16 in glomerular diseases (Figure 5).

\section{Discussion}


In this study, we demonstrated that podocyte TGF- $\beta 1$ could affect the behavior of p16 in glomerular endothelial cells in vivo and in vitro. We also clarified the pathological and clinical phenotypic changes in kidneys induced by the practical level of TGF- $\beta 1$ expression.

The most important finding in this study is that TGF- $\beta 1$ was involved in senescence-related pathways via not only $\mathrm{p} 21$ but also $\mathrm{p} 16$ in glomeruli in vivo and in vitro, since p16 and p21 are major responsible molecules for cellular senescence. The relationship between TGF- $\beta 1$-Smad3 pathway and p21 has been well clarified ${ }^{19,20,21,22}$. However, the direct effect of TGF- $\beta 1$-Smad3 pathway on $\mathrm{p} 16$ behavior has not been investigated, especially in vivo. We revealed the novel mechanism of TGF- $\beta 1$ involvement in $p 16$ behavior, which was different from the interaction between TGF- $\beta 1$ and p21. TGF- $\beta 1$ could not increase p16 expression in glomeruli, but could induce nuclear translocation of p16 in glomerular endothelial cells.

p16 expression in kidney tubules and interstitial cells has been studied in mouse models and human kidney diseases in the progression of kidney 
fibrosis and aging kidney ${ }^{29}$. For example, high phosphate activates senescence in kidney tubular cells through distinct but interconnected mechanisms: upregulating p16/p21, and elevating plasminogen activator inhibitor-1 and downregulating Klotho, followed by fibrosis ${ }^{30}$. In addition, the acute kidney injury-to-chronic kidney disease transition may involve a wide range of mechanisms including scar-forming myofibroblasts, microvascular rarefaction, mitochondrial dysfunction, or cell cycle arrest by the involvement of epigenetic, gene, and protein alterations leading to common final signaling pathways such as TGF- $\beta 1, \mathrm{p} 16, \mathrm{Wnt} / \beta$-catenin pathway involved in renal aging ${ }^{31}$. However, to our knowledge, p16 expression in each cell comprised of glomeruli has not been fully investigated. In human kidney diseases such as IgA nephropathy, nephrotic syndrome, and diabetic kidney disease as well as aging kidney, p16 expression was found in mesangial, endothelial cells and podocytes ${ }^{5,6,7,8}$. On the other hand, there were few reports evaluating p16 expression in glomeruli using the in vivo animal kidney disease model. Aratani et al. showed that p16 is involved in radiation-induced kidney disease by immunohistochemical analysis ${ }^{32}$. In the 
diabetic kidney disease model, p16 expression increased significantly in glomeruli by western blot analysis $33,34,35$. So far, our animal study is the first one to evaluate p16 expression in glomeruli quantitatively by both western blot and immunohistochemical analysis in vivo. We could reveal that the increase in nuclei positive immunostaining of p16 does not always coincide with the upregulation of $\mathrm{p} 16$ expression in vivo.

There were several previous papers which reported the role of TGF- $\beta 1$ by using the TGF- $\beta 1$ overexpression mouse model as described in "Introduction". Kopp et al. reported that TGF- $\beta 1$ overexpression in liver can cause kidney glomerulosclerosis ${ }^{14}$. However, this model has an eight times higher expression level. Hathaway et al. revealed that TGF- $\beta 1$ expression level can influence the kidney manifestation in the mice, especially under diabetic conditions ${ }^{13}$. These findings mean that TGF- $\beta 1$ can cause glomerulosclerosis if its concentration is extremely high or any other risk factors such as cytokines and metabolic conditions concur with TGF- $\beta 1$ stimulation. In human kidney diseases, the reports evaluating plasma TGF- $\beta 1$ concentration were limited. Plasma TGF- $\beta 1$ 
concentration can increase according to kidney dysfunction or diabetic kidney injury. In older community dwelling adults, the levels of median plasma TGF- $\beta 1$ were higher for those with eGFR $<60 \mathrm{ml} / \mathrm{min} / 1.73 \mathrm{~m}^{2}$ compared to those with eGFR $>60 \mathrm{ml} / \mathrm{min} / 1.73 \mathrm{~m}^{2}{ }^{36}$. In patients with diabetic kidney disease, baseline median plasma TGF- $\beta 1$ levels were two times higher in participants with progressive kidney disease compared to participants whose kidney disease had not progressed ${ }^{37}$. Iwano et al. investigated intraglomerular TGF- $\beta 1$ mRNA in patients with human kidney disease. TGF- $\beta 1$ mRNA was significantly elevated in patients with mesangial proliferative glomerulonephritis having a moderate increase in mesangial matrix, diabetic nephropathy and lupus nephritis compared to participants with normal glomeruli. Levels of TGF- $\beta 1$ mRNA expression in patients with diffuse proliferative lupus nephritis were more than five times higher than those with normal glomeruli38. In our mouse model, unfortunately, we could not estimate the local expression level of total TGF- $\beta 1$ in glomeruli quantitatively, because we used an overexpression model of porcine TGF- $\beta 1$, which does not have the same potency as mouse TGF- $\beta 1$. However, 
our mice had similar plasma TGF- $\beta 1$ concentration as the control mice, which is consistent with the previous report using the same mice ${ }^{39}$. Therefore, we think that the meaning of our mouse model is to clarify the basic background lesion in various human kidney diseases, because TGF- $\beta 1$ is involved in the development and progression of these diseases ${ }^{9,10}$. Our mice would represent the early stage of glomerular lesion considering pathological changes such as mild mesangial expansion, podocyte injury and albuminuria. Moreover, we could show the practical level of TGF- $\beta 1$ per se cause the expression of senescence-related molecules in the nuclei of glomerular endothelial cells. Therefore, we believe endothelial senescence can be triggered in the early stage of various human kidney diseases, because we could find the expression of p16 in the nuclei of endothelial cells in human renal biopsy samples from many kinds of kidney diseases in this study (Figure 5), which was consistent with the previous findings of glomerular TGF- $\beta 1$ mRNA expression in patients with kidney diasease ${ }^{38}$. Endothelial senescence could be one of the important mechanisms in the progression of arteriosclerosis in glomeruli ${ }^{19}$. Probably, in addition to the 
TGF- $\beta$ 1-related basic alterations of pathology and molecular behavior in mesangial, endothelial cells and podocytes shown in this study, various cytokines and growth factors modify kidney lesions, followed by the establishment of complex and disease-specific kidney manifestation.

In this study, we investigated the phenotype in podocyte-specific TGF- $\beta 1$ overexpression mice. Regarding the expression site of TGF- $\beta 1$ in the glomeruli of human kidney diseases, both Yamamoto et al. and Ito et al. reported that TGF- $\beta 1$ is expressed in podocytes as well as mesangial, endothelial cells of glomeruli in patients with proliferative nephritis ${ }^{40,41}$. In patients with advanced diabetic nephropathy, TGF- $\beta 1$ is immunostained in both matrix and remnant cells of glomeruli ${ }^{42,43}$. Our mouse model can partly explain the pathogenic role of TGF- $\beta 1$ in these glomerulopathies. In addition, considering the phenotype of the mice having TGF- $\beta 1$ overexpression in glomerular endothelial cells for 28 days in the previous report ${ }^{15}$, which resembled our results in terms of podocyte injury and proteinuria, TGF- $\beta 1$ could cause a podocyte-endothelial crosstalk ${ }^{44}$. 
In conclusion, we could find the TGF- $\beta 1$-Smad3 pathway involvement in the behavior of p16 in glomeruli in vivo and in vitro. These findings will be one of the common and novel mechanisms in the progression of various human kidney diseases.

\section{Methods}

\section{Ethics statement}

All clinical investigations were conducted according to the principles

expressed in the Declaration of Helsinki. All patients gave their informed, written consent to participate and publish. The animal experiment was carried out in compliance with the ARRIVE guidelines. All experiments were performed in accordance with the institutional guidelines and regulations of Tokushima University. The study including animal experiments was approved by the Research Ethics Committee of Tokushima University. 


\section{Subjects}

Renal biopsy samples derived from different human glomerular disease such as minimal change disease, lupus nephritis, IgA nephropathy, purpura nephritis, membranous proliferative glomerulonephritis, membranous nephropathy, ANCA glomerulonephritis, and diabetic nephropathy diagnosed at Tokushima University Hospital were analyzed in this study. Renal biopsy tissues were fixed in Dubosque-Brazil's solution. Three controls consisted of biopsies from patients with asymptomatic hematuria. They showed minor glomerular abnormalities and negative immunofluorescence. The profiles of control and patients with human kidney disease are shown in Table 1.

Mice

Podocin-Cre mice, Cre-dependent HA-tagged TGF- $\beta 1$ overexpression mice were obtained from The Jackson Laboratory (Bar Harbor, ME, USA). All mice were 10 times backcrossed to ICR (CLEA Japan, Inc., Tokyo, Japan) before starting this experiment. In PodCre(+) TGF mice, HA-tag was conjugated with 
bioactive porcine TGF- $\beta 1$. Urine and plasma were collected from the mice and they were sacrificed at one year of age to analyze the pathological changes in the kidney.

\section{Immunohistochemical analysis}

Immunohistochemical analysis was performed on paraffin embedded

sections by indirect immunohistochemistry procedure using rabbit anti-p16

(sc-1207, Santa Cruz Biotechnology, Dallas, TX, USA), anti-p21 (ab109199,

Abcam plc, Cambridge, UK), anti-HA (3724, Cell Signaling Technology, Beverly, MA, USA), anti-CD34 (ab81289, abcam), goat anti-collagen IV (1340-01,

SouthernBiotech, Birmingham, AL, USA) and sheep anti-nephrin (AF4269, R\&D

Systems, Minneapolis, MN, USA) antibodies. For p16, p21 and collagen IV

immunostaining, sections were pretreated by proteinase K (19131, QIAGEN

K.K., Tokyo, Japan). For the other antigens, sections were pretreated by citrate

buffer ( $\mathrm{pH}$ 6.0). Following the first antibody, sections were incubated with Alexa

Fluor 488 or 594-conjugated donkey anti-rabbit antibody (A21206, A21207, 
Invitrogen, Grand Island, NY, USA) for p16, p21, HA, and Alexa Fluor 488-conjugated donkey anti-goat antibody (A11055, Invitrogen) for collagen IV and Alexa Fluor 488-conjugated donkey anti-sheep antibody (A11015, Invitrogen) for nephrin. Nuclei were visualized by DAPI (D523, DOJINDO Laboratories, Kumamoto, Japan). For CD34 immunostaining, Avidin/Biotin Blocking System (SIG-31126, BioLegend, Inc., San Diego, CA, USA), biotin-conjugated anti-rabbit antibody, HRP-conjugated streptavidin (426011, 426061, Nichirei Biosciences Inc., Tokyo, Japan) and DAB substrate kit (SK-4100, Vector Laboratories, Inc., Burlingame, CA, USA) were used. The immunohistochemical signal was quantified using Image $\mathrm{J}^{25}$. The values were expressed as a percentage of glomerular surface area occupied by immunostained area for collagen IV immunostained area. The positively stained nuclei number was counted for $\mathrm{p} 16$ and p21 immunostaining. The mean values of the data obtained from six to eight mice were shown. For each sample, 25 glomerular profiles were measured. In human renal biopsy samples, 10 glomerular profiles per each subject were analyzed. 


\section{Senescence associated $\beta$-galactosidase activity}

Senescence associated $\beta$-galactosidase was detected using

Senescence $\beta$-Galactosidase Staining Kit (9860, Cell Signaling Technology)

according to the manufacturer's instruction. For each sample, 25 glomerular

profiles were measured. The mean values of the data obtained from three to four mice were shown.

\section{Western blotting}

In vivo, mouse glomeruli at one year of age were collected by magnetic beads-based isolation ${ }^{26}$. Briefly, the transcardiac perfusion was performed with phosphate buffered saline containing precleaned beads (Dynabeads, Invitrogen). The perfused renal cortex was briefly digested with collagenase A (Roche, Basel, Switzerland) and deoxyribonuclease I (Invitrogen), and the glomeruli stuffed with beads were isolated by DYNAL (Invitrogen). Glomeruli were lysed using Mammalian Cell Extraction Kit (BioVision, Inc., Milpitas, CA, USA). Lysates of 
glomeruli were applied to SDS-PAGE and immunoblotted with the following primary antibodies: rabbit antibody against p16 (ab108439, abcam), p21 (ab109199, abcam), phospho-Smad3 (ab52903, abcam), Smad3 (ab28379, abcam), and mouse $\alpha$-tubulin (T6199, Sigma-Aldrich, St. Louis, MO, USA). In vitro, lysates of cultured endothelial cells were immunoblotted with the antibodies mentioned above and goat anti-Histon H3 (sc-8654, Santa Cruz Biotechnology). Immobilon ECL Ultra Western HRP Substrate (Merck Millipore, Billerica, MA, USA) was used to detect the blotting signals using LAS-3000 (FUJIFILM, Tokyo, Japan). The immunohistochemical signal was quantified using Image $\mathrm{J}^{25}$. The mean values of the data obtained from four to six mice or three to four independent in vitro experiments were shown.

\section{Electron microscopy}

Tissues used for electron microscopy were fixed with $2.5 \%$

glutaraldehyde. We entrusted electron microscopy analysis to a specialized company (BML, Inc. Tokyo, Japan.) ${ }^{27}$. Glomerular basement membrane width 
was measured using Image $\mathrm{J}^{25}$. The mean values of the data obtained from three to four mice were shown. For each sample, six glomerular basement membrane widths were measured.

\section{Urine albumin and creatinine}

Urinary albumin and creatinine were determined using Albuwell M kit and Creatinine Companion kit (Exocell Inc., Philadelphia, PA, USA).

\section{Plasma TGF- $\beta 1$ concentration}

Mouse plasma was obtained using heparinized hematocrit tubes

(Drummmond scientific company, Broomall, PA, USA). Plasma TGF- $\beta 1$

concentration was analyzed by quantikine ELISA ( $R$ and $D$ systems).

\section{Endothelial cell culture}

Mouse immortalized endothelial cell line, TKD2 (RIKEN BioResource

Research Center, Ibaraki, Japan) was maintained in growth medium (Dulbecco's 
modified Eagle's medium) (Sigma) supplemented with $1 \mathrm{mM}$ glutamine, penicillin at $100 \mathrm{units} / \mathrm{ml}$, streptomycin at $100 \mu \mathrm{g} / \mathrm{ml}$ (Invitrogen) and $10 \%$ fetal bovine serum (Sigma) at 33 degrees centigrade. The cells $\left(1.2 \times 10^{6} /\right.$ well) were plated in 9-cm culture dishes (Fine Plus International Ltd., Kyoto, Japan). Twenty-four hours later, the cells were serum-starved in DMEM containing $0.5 \%$ bovine serum albumin (Sigma) and pretreated with DMSO (Sigma) or SB431542 $(1 \mu \mathrm{M})$, a potent and specific inhibitor of TGF- $\beta$ type I receptor (Cayman Chemical, Michigan, USA), for two hours. They were stimulated with TGF- $\beta 1$ (10 ng/ml) (PeproTech, Rocky Hill, NJ, USA). Cell lysates were harvested using Mammalian Cell Extraction Kit or Nuclear/Cytosol Fractionation Kit (BioVision, Inc., Milpitas, CA, USA), 30 minutes or 24 hours after stimulation. In a transfection experiment, control or constitutively active Smad3 expression vector was kindly provided by Dr. J. Oh (Korea University) ${ }^{28}$. Plasmids were transfected using Novagen $\circledast$ GeneJuice ${ }^{\circledR}$ Transfection Reagents (Merck millipore) according to the manufacturer's protocol. Cell lysates were harvested 24 hours after transfection. 


\section{Statistical Analysis}

All values are expressed as mean \pm SD. Statistical analysis was

performed using SPSS for Windows version 13.0 (SPSS, Inc., Chicago, IL, USA).

The results were compared using student's t-test or Welch's t-test, if data were normally distributed. Non-normal data were analyzed by Man-Whitney's U test. F-test was used for comparing the factors of total deviation. Significance was defined by $P$ less than 0.05 .

\section{References}

1. McHugh, D. \& Gil, J. Senescence and aging: Causes, consequences, and therapeutic avenues. J Cell Biol. 217, 65-77 (2018).

2. Kuilman, T., Michaloglou, C., Mooi, W.J. \& Peeper, D.S. The essence of senescence. Genes Dev. 24, 2463-2479 (2010) 
3. Salama, R., Sadaie, M., Hoare, M. \& Narita, M. Cellular senescence and its effector programs. Genes Dev. 28, 99-114 (2014)

4. Idda, M.L. et al. Survey of senescent cell markers with age in human tissues. Aging (Albany NY). 12, 4052-4066 (2020)

5. Melk, A. et al. Expression of p16INK4a and other cell cycle regulator and senescence associated genes in aging human kidney. Kidney Int. 65, 510-520 (2004)

6. Yamada, K. et al. Expression of age-related factors during the development of renal damage in patients with IgA nephropathy. Clin Exp Nephrol. 19, 830-837 (2015)

7. Sis, B. et al. Accelerated expression of senescence associated cell cycle inhibitor p16INK4A in kidneys with glomerular disease. Kidney Int. 71, 218-226 (2007)

8. Verzola, D. et al. Accelerated senescence in the kidneys of patients with type 2 diabetic nephropathy. Am J Physiol Renal Physiol. 295, F1563-1573 (2008) 
9. Yamamoto, T. et al. Expression of transforming growth factor-beta isoforms in human glomerular diseases. Kidney Int. 49, 461-469 (1996)

10. Branton, M.H. \& Kopp, J.B. TGF-beta and fibrosis. Microbes Infect. 1, $1349-1365$ (1999)

11. Schnaper, H.W. et al. TGF-beta signal transduction in chronic kidney disease. Front Biosci (Landmark Ed). 14, 2448-2465 (2009)

12. Meng, X.M., Nikolic-Paterson, D.J. \& Lan, H.Y. TGF- $\beta$ : the master regulator of fibrosis. Nat Rev Nephrol. 12, 325-338 (2016)

13. Hathaway, C.K. et al. Low TGF $\beta 1$ expression prevents and high expression exacerbates diabetic nephropathy in mice. Proc Natl Acad Sci U S A. 112, $5815-5820(2015)$

14. Kopp, J.B. et al. Transgenic mice with increased plasma levels of TGF-beta 1 develop progressive renal disease. Lab Invest. 74. 991-1003 (1996)

15. Ghayur, A. et al. Adenovirus-mediated gene transfer of TGF- $\beta 1$ to the renal glomeruli leads to proteinuria. Am J Pathol. 180, 940-951 (2012) 
16. Lyu, G. et al. TGF- $\beta$ signaling alters H4K20me3 status via miR-29 and contributes to cellular senescence and cardiac aging. Nat Commun. 9, 2560 (2018)

17. Morikawa, M., Derynck, R. \& Miyazono, K. TGF- $\beta$ and the TGF- $\beta$ Family: Context-Dependent Roles in Cell and Tissue Physiology. Cold Spring Harb Perspect Biol. 8, a021873 (2016)

18. Tominaga, K. \& Suzuki, H.I. TGF- $\beta$ Signaling in Cellular Senescence and Aging-Related Pathology. Int J Mol Sci. 20, 5002 (2019)

19. Samarakoon, R., Higgins, S.P., Higgins, C.E. \& Higgins, P.J. The TGF-ק1/p53/PAI-1 Signaling Axis in Vascular Senescence: Role of Caveolin-1.

Biomolecules. 9, 341 (2019)

20. Moustakas, A. \& Kardassis, D. Regulation of the human p21/WAF1/Cip1 promoter in hepatic cells by functional interactions between Sp1 and Smad family members. Proc Natl Acad Sci U S A. 95, 6733-6738 (1998) 
21. Pardali, K. et al. Role of Smad proteins and transcription factor Sp1 in p21(Waf1/Cip1) regulation by transforming growth factor-beta. J Biol Chem. 275, 29244-29256 (2000)

22. Moustakas, A., Pardali, K., Gaal, A. \& Heldin, C.H. Mechanisms of TGF-beta signaling in regulation of cell growth and differentiation. Immunol Lett. 82, 85-91 (2002)

23. Shimoda, H. et al. Inhibition of the H3K4 methyltransferase MLL1/WDR5 complex attenuates renal senescence in ischemia reperfusion mice by reduction of p16 INK4a. Kidney Int. 96, 1162-1175 (2019)

24. Kandhaya-Pillai, R. et al. SMAD4 mutations and cross-talk between TGF- $\beta / \mathrm{IFNY}$ signaling accelerate rates of DNA damage and cellular senescence, resulting in a segmental progeroid syndrome-the Myhre syndrome.

Geroscience. doi: 10.1007/s11357-020-00318-6 (2021)

25. Schneider, C.A., Rasband, W.S. \& Eliceiri, K.W. NIH Image to Image J: 25 years of image analysis. Nat Methods. 9, 671-675 (2012) 
26. Takemoto, M. et al. A new method for large scale isolation of kidney glomeruli from mice. Am J Pathol. 161, 799-805 (2002) 27. Fujita, Y. et al. An adjustment in BMP4 function represents a treatment for diabetic nephropathy and podocyte injury. Sci Rep. 8, 13011 (2018) 28. Ono, H. et al. Novel Interplay Between Smad1 and Smad3 Phosphorylation via AGE Regulates the Progression of Diabetic Nephropathy. Sci Rep. 8, 10548 (2018)

29. Naesens, M. Replicative senescence in kidney aging, renal disease, and renal transplantation. Discov Med. 11, 65-75 (2011)

30. Maique, J. et al. High Phosphate Induces and Klotho Attenuates Kidney Epithelial Senescence and Fibrosis. Front Pharmacol. 11, 1273 (2020) 31. Franzin, R. et al. Inflammaging and Complement System: A Link Between Acute Kidney Injury and Chronic Graft Damage. Front Immunol. 11:734 (2020) 32. Aratani, S. et al. Radiation-induced premature cellular senescence involved in glomerular diseases in rats. Sci Rep. 8, 16812 (2018) 
33. del Nogal, M. et al. Hyperosmolarity induced by high glucose promotes senescence in human glomerular mesangial cells. Int J Biochem Cell Biol. 54, 98-110 (2014)

34. Wolf, G., Wenzel, U., Ziyadeh, F.N. \& Stahl, R.A. Angiotensin converting-enzyme inhibitor treatment reduces glomerular p16INK4 and p27Kip1 expression in diabetic BBdp rats. Diabetologia. 42, 1425-1432 (1999) 35. Zhang, Y.Y. et al. p16ink4a Expression Is Increased through 12-Lipoxygenase in High Glucose-Stimulated Glomerular Mesangial Cells and Type 2 Diabetic Glomeruli. Nephron. 130, 141-150 (2015)

36. Mehta, T. et al. Higher plasma transforming growth factor (TGF)- $\beta$ is associated with kidney disease in older community dwelling adults.

BMC Nephrol. 18, 98 (2017)

37. Wong, M.G. et al. Circulating bone morphogenetic protein-7 and transforming growth factor- $\beta 1$ are better predictors of renal end points in patients with type 2 diabetes mellitus. Kidney Int. 83, 278-284 (2013) 
38. Iwano, M. et al. Intraglomerular expression of transforming growth factor-beta 1 (TGF-beta 1) mRNA in patients with glomerulonephritis: quantitative analysis by competitive polymerase chain reaction. Clin Exp Immunol. 97, 309-314 (1994)

39. Hall, B.E. et al. Conditional overexpression of TGF-beta1 disrupts mouse salivary gland development and function. Lab Invest. 90, 543-555 (2010) 40. Yamamoto, T. et al. Expression of transforming growth factor-beta isoforms in human glomerular diseases. Kidney Int. 49, 461-469 (1996) 41. Ito, Y. et al. Expression patterns of connective tissue growth factor and of TGF-beta isoforms during glomerular injury recapitulate glomerulogenesis. $\mathrm{Am} J$ Physiol Renal Physiol. 299, F545-558 (2010)

42. Yamamoto, T., Nakamura, T., Noble, N.A., Ruoslahti. E. \& Border, W.A. Expression of transforming growth factor beta is elevated in human and experimental diabetic nephropathy. Proc Natl Acad Sci U S A. 90, 1814-1818 (1993) 
43. Shankland, S.J., Scholey, J.W., Ly, H. \& Thai, K. Expression of transforming growth factor-beta 1 during diabetic renal hypertrophy. Kidney Int. 46, 430-442

44. Eremina, V., Baeld, H.J. \& Quaggin, S.E. Role of the VEGF--a signaling pathway in the glomerulus: evidence for crosstalk between components of the glomerular filtration barrier. Nephron Physiol. 106, 32-37 (2007)

\section{Acknowledgements}

This work was supported by funds from Grant-in-Aid for Research for

Scientific Research 16K09619 and 19K08705. We thank Dr. Hideharu Abe

(Tokushima University) for his valuable advice to perform this study, and Dr. J.

Oh (Korea University) for the gift of the constitutively active Smad3 expression vector. We also thank Shinobu Kajino, Akiyo Muramoto (Tokushima University) for their clinical assistance, and Masashi Miyoshi, Hisae Nakagawa, Sanae Hayashi (Tokushima University) for their technical assistance. 


\section{Authors' contributions statement}

S.U. and T.T. conducted the experiments. A.O. and A.S. analyzed the

data. K.Nishimura. and E.S. acquired the clinical data. S.W. gave us valuable

advises to this manuscript. M.T. supervised the manuscript. K.Nagai. conceived

the experiments, interpreted the data and drafted the manuscript. All authors

reviewed the manuscript.

Additional information

Competing interests

The authors declare no competing interests. 
Figure 1
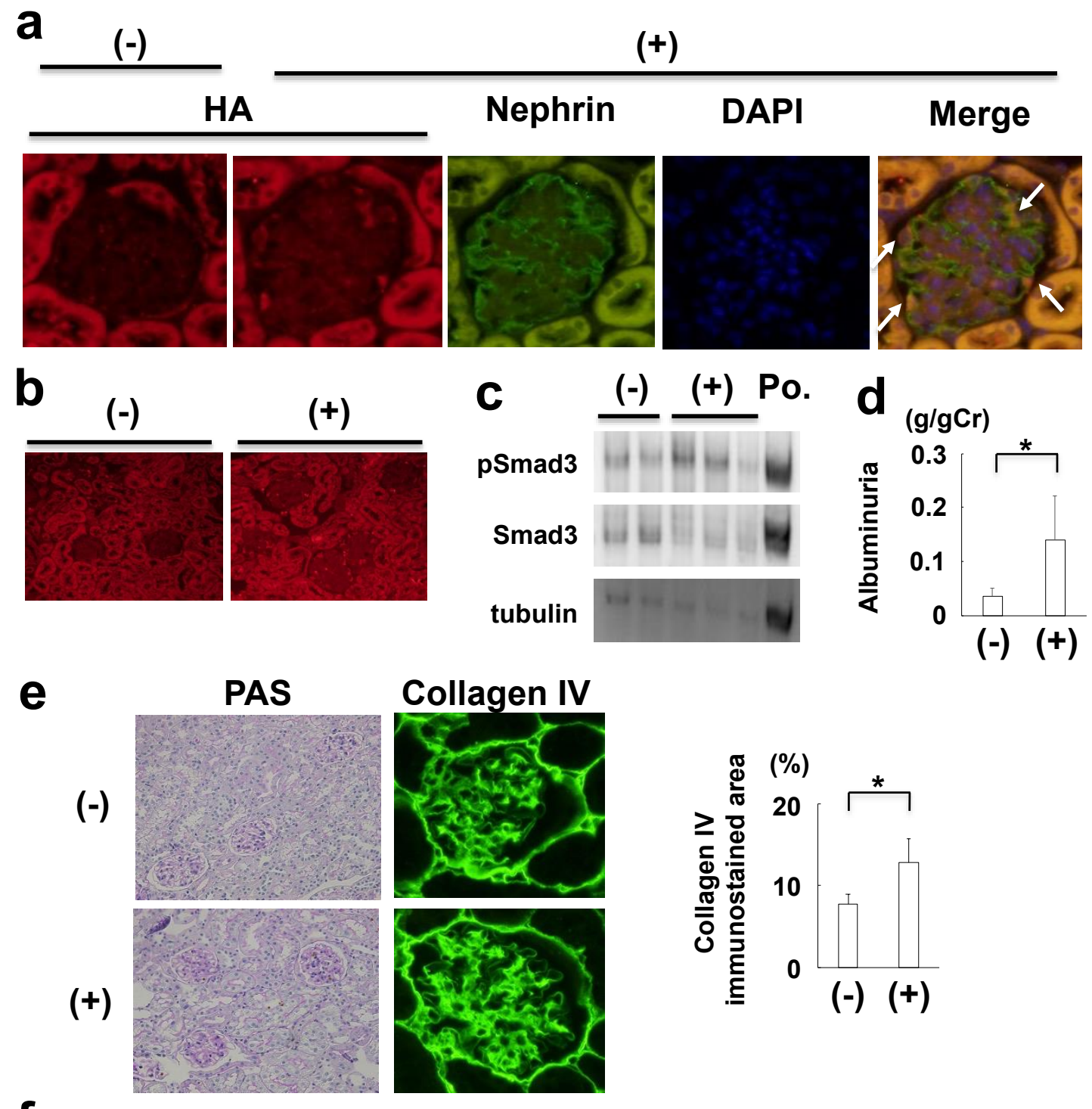

f

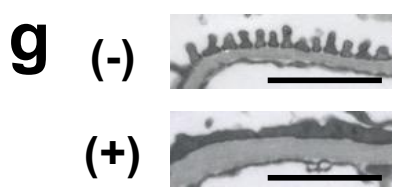

(-)
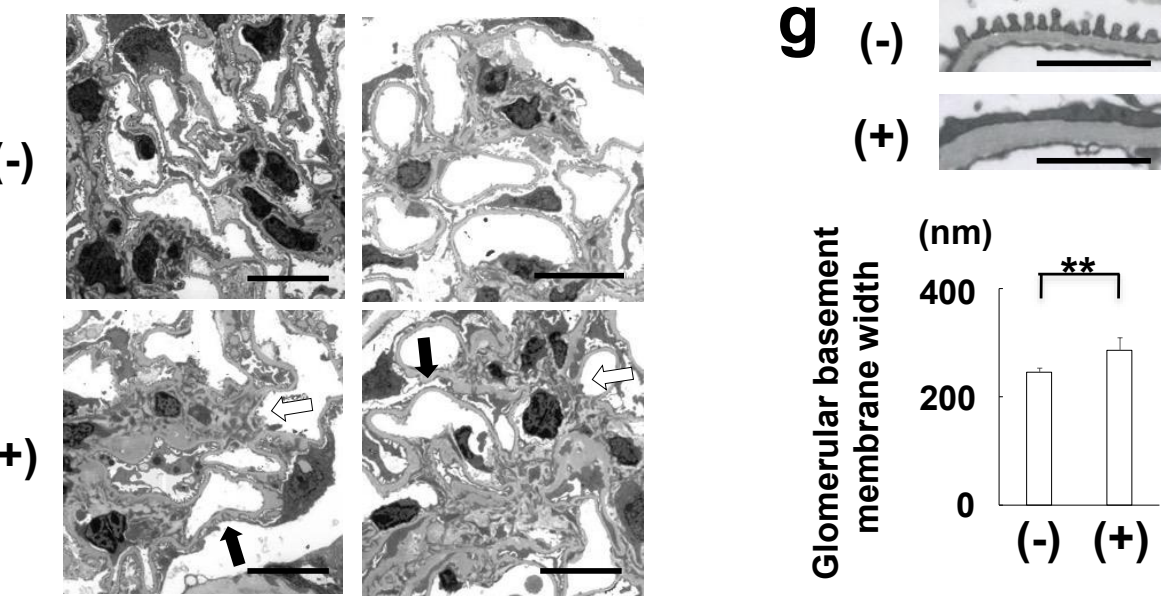
Figure 1. Characterization and pathological changes of podocyte-specific TGF- $\beta 1$ overexpression mice.

(a) TGF- $\beta 1$ was expressed in podocytes. HA-tag was conjugated with bioactive porcine TGF- $\beta 1$ in PodCre(+) TGF mice. HA-tag merged with nephrin (podocyte marker). (b) Diffuse expression of HA-tag conjugated TGF- $\beta 1$ was observed in the kidney of PodCre(+) TGF mice. (c) Representative pictures of western blot analysis of glomeruli protein. Smad3 was phosphorylated in glomeruli in PodCre(+) TGF mice. (d) Urine albumin excretion was significantly increased in PodCre(+) TGF mice. ( $\mathrm{N}=6$ in control mice, $\mathrm{N}=8$ in PodCre(+) TGF mice). ${ }^{*} P$ $<0.01$ (Man-Whitney's U test). (e) Representative pictures of periodic acid-schiff (PAS) stain and collagen IV immunohistochemistry in PodCre(+) TGF mice. PodCre(+) TGF mice showed a significant increase in collagen IV immunostained area ( $\mathrm{N}=6$ in control mice, $\mathrm{N}=8$ in PodCre (+) TGF mice). ${ }^{*} P$ $<0.01$ (t-test). (f) Representative pictures of electron microscopy. PodCre $(+)$ TGF mice showed mesangial expansion (white arrow) and diffuse foot process effacement (black arrow). Scale bar: $10 \mu \mathrm{m}$. (g) Representative pictures of 
glomerular basement membrane by using electron microscopy and quantitative evaluation of glomerular basement membrane width. PodCre(+) TGF mice showed a significant thickening of glomerular basement membrane $(\mathrm{N}=3$ in control mice, $\mathrm{N}=4$ in PodCre(+) TGF mice). Scale bar: $2 \mu \mathrm{m} .{ }^{* \star} P<0.05$ (t-test). (-): Control mice. (+): Podocyte-specific TGF- $\beta 1$ overexpression mice (PodCre(+) TGF mice). Po.: positive control. pSmad3: phosphorylated Smad3. n.s.: not significant. 
Figure 2

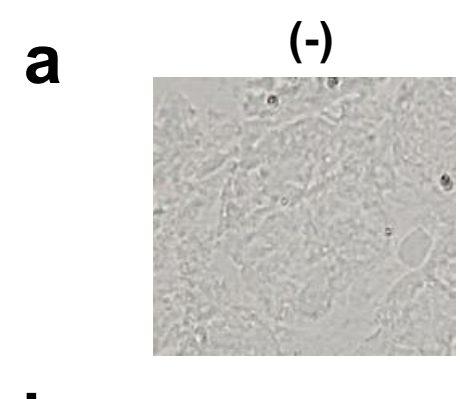

b

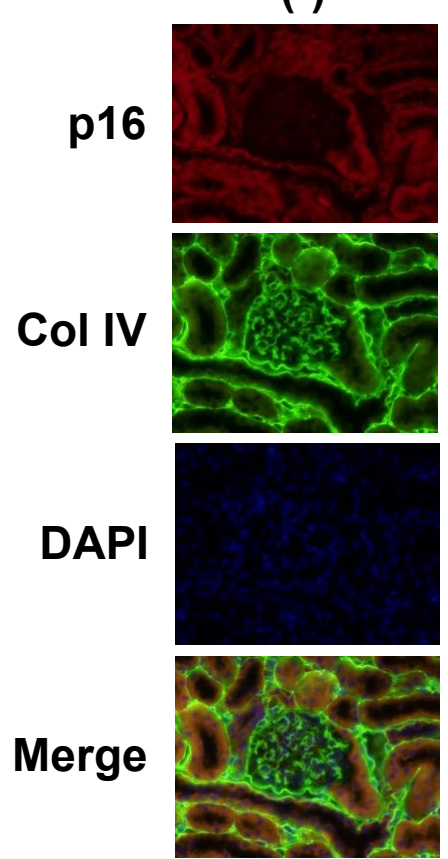

Endo.

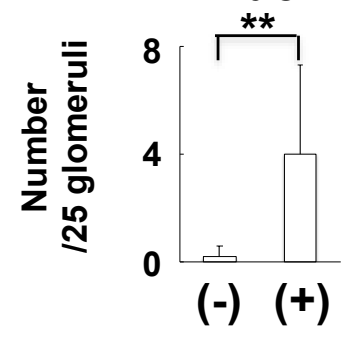

(+)

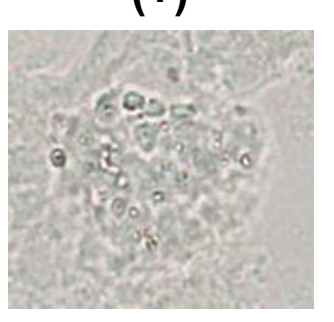

(+)
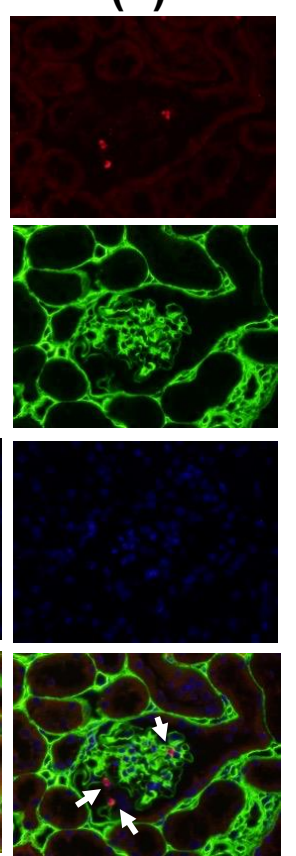

Mes.

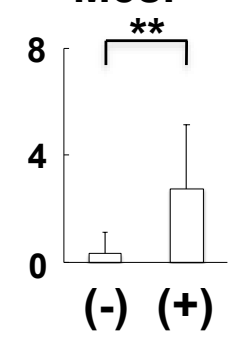

C

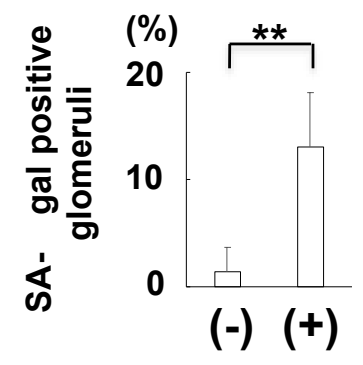

$(-) \quad(+)$

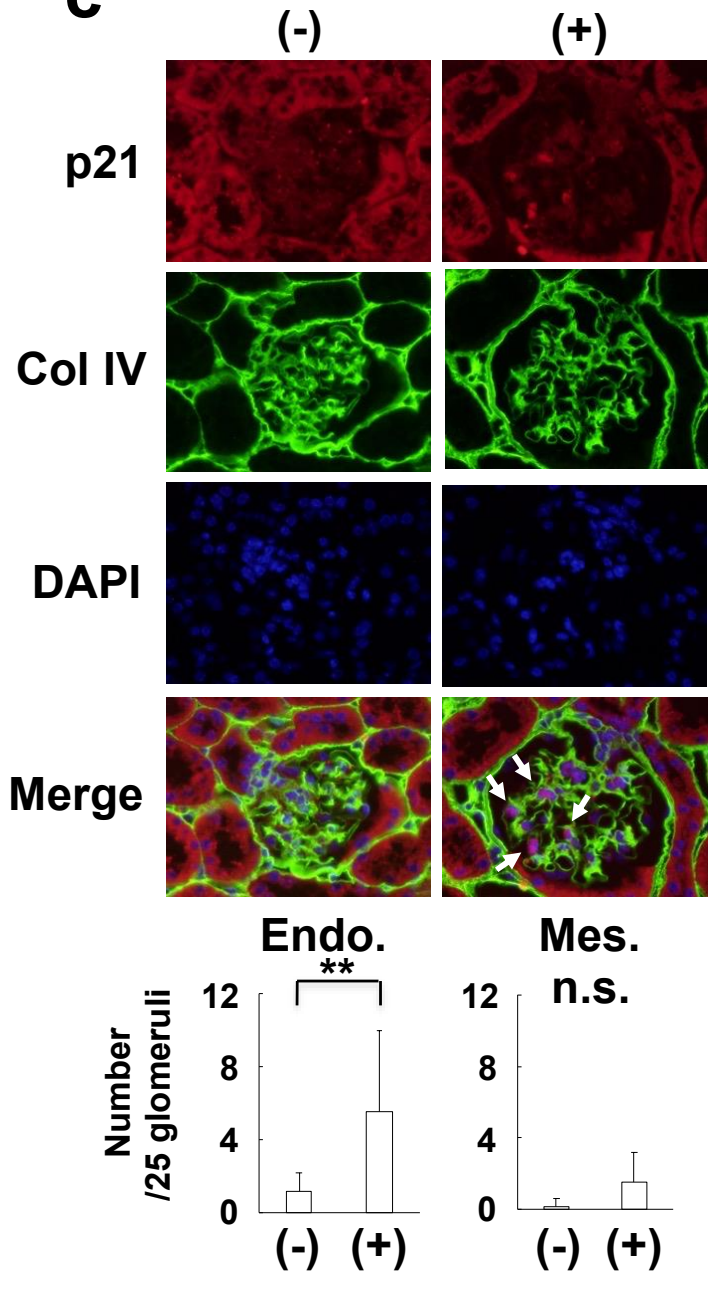

d

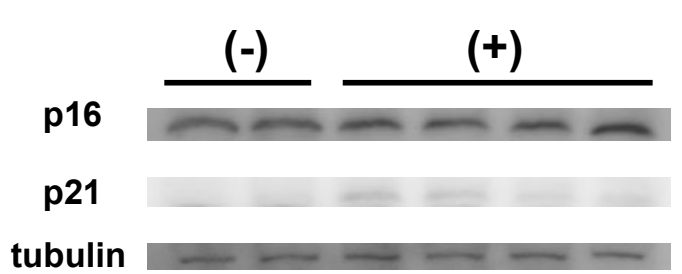

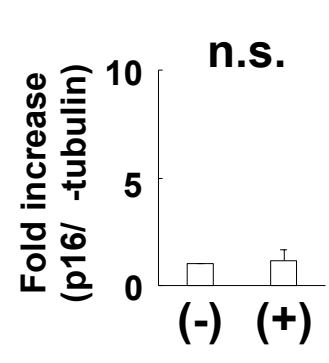

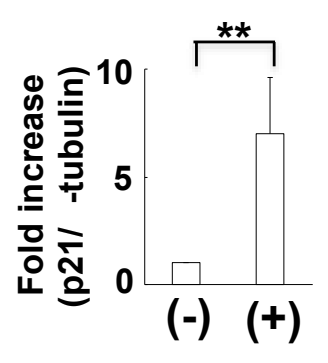


Figure 2. Detection of the markers for cellular senescence in podocyte-specific TGF- $\beta 1$ overexpression mice.

(a) Representative pictures of senescence-associated $\beta$-galactosidase staining. Senescence-associated $\beta$-galactosidase activity was significantly increased in PodCre(+) TGF mice. $\left(\mathrm{N}=3\right.$ in control mice, $\mathrm{N}=4$ in PodCre(+) TGF mice). ${ }^{* \star} P$ $<0.05$ (t-test). (b) Representative pictures of p16 immunohistochemistry. PodCre(+) TGF mice had p16 expression mainly in endothelial cells. PodCre $(+)$ TGF mice showed a significant increase in p16 immunostained nuclei in endothelial and mesangial cells. ( $N=6$ in control mice, $N=8$ in PodCre(+) TGF mice). ${ }^{* *} P<0.05$ (t-test). (c) Representative pictures of p21 immunohistochemistry. PodCre(+) TGF mice had p21 expression mainly in endothelial cells. PodCre(+) TGF mice showed a significant increase in p21 immunostained nuclei in endothelial cells. ( $N=6$ in control mice, $N=8$ in PodCre(+) TGF mice). ${ }^{* *} P<0.05$ (t-test). (d) Representative pictures of western blot analysis of $\mathrm{p} 16$ and p21 expression in glomeruli. PodCre(+) TGF mice had a 
significant expression of $\mathrm{p} 21$, but not that of $\mathrm{p} 16 .(\mathrm{N}=4$ in control mice, $N=6$ in

PodCre(+) TGF mice). ${ }^{* *} P<0.05$ (t-test).

(-): Control mice. (+): Podocyte-specific TGF- $\beta 1$ overexpression mice

(PodCre(+) TGF mice). SA- $\beta$-gal: Senescence-associated $\beta$-galactosidase.

Col IV: Collagen IV. Endo.: Endothelial cell. Mes.: Mesangial cell. n.s.: not

significant. 
Figure 3

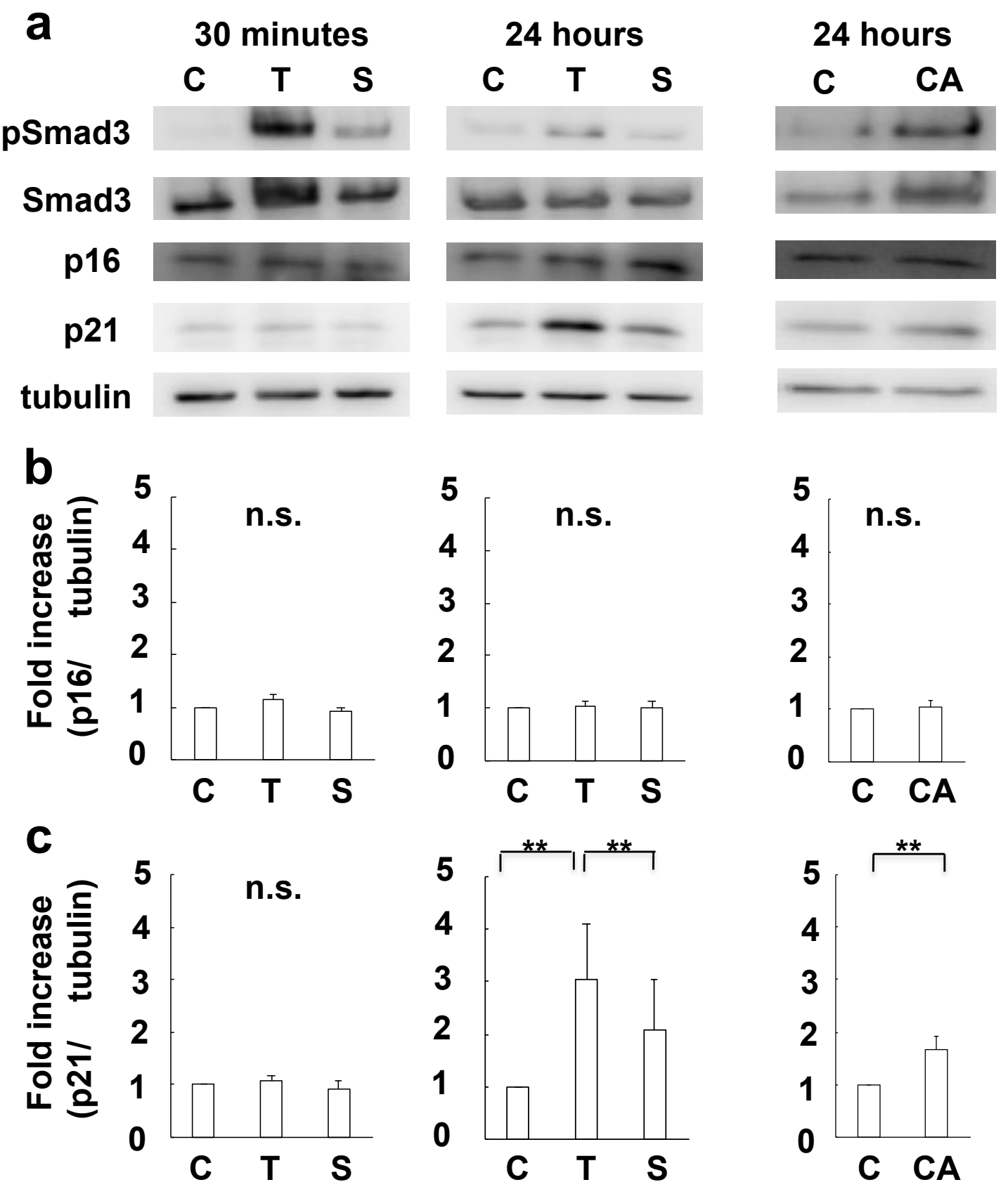

Figure 3. Expression of p16 and p21 induced by the activation of

TGF- $\beta 1-S m a d 3$ pathway in endothelial cells. 
(a) Representative pictures of western blot analysis of p16 and p21 expression in endothelial cells induced by the stimulation of TGF- $\beta 1$-Smad3 pathway. (b, c) Activation of TGF- $\beta 1$-Smad3 pathway can increase the expression of p21 in 24 hours (late phase), but not that of p16 in endothelial cells $(\mathrm{N}=3)$.

C: Control. T: TGF- $\beta 1$. S: SB431542. CA: constitutive active Smad3. pSmad3: phosphorylated Smad3. n.s.: not significant. ${ }^{* *} P<0.05$ (t-test). 
Figure 4

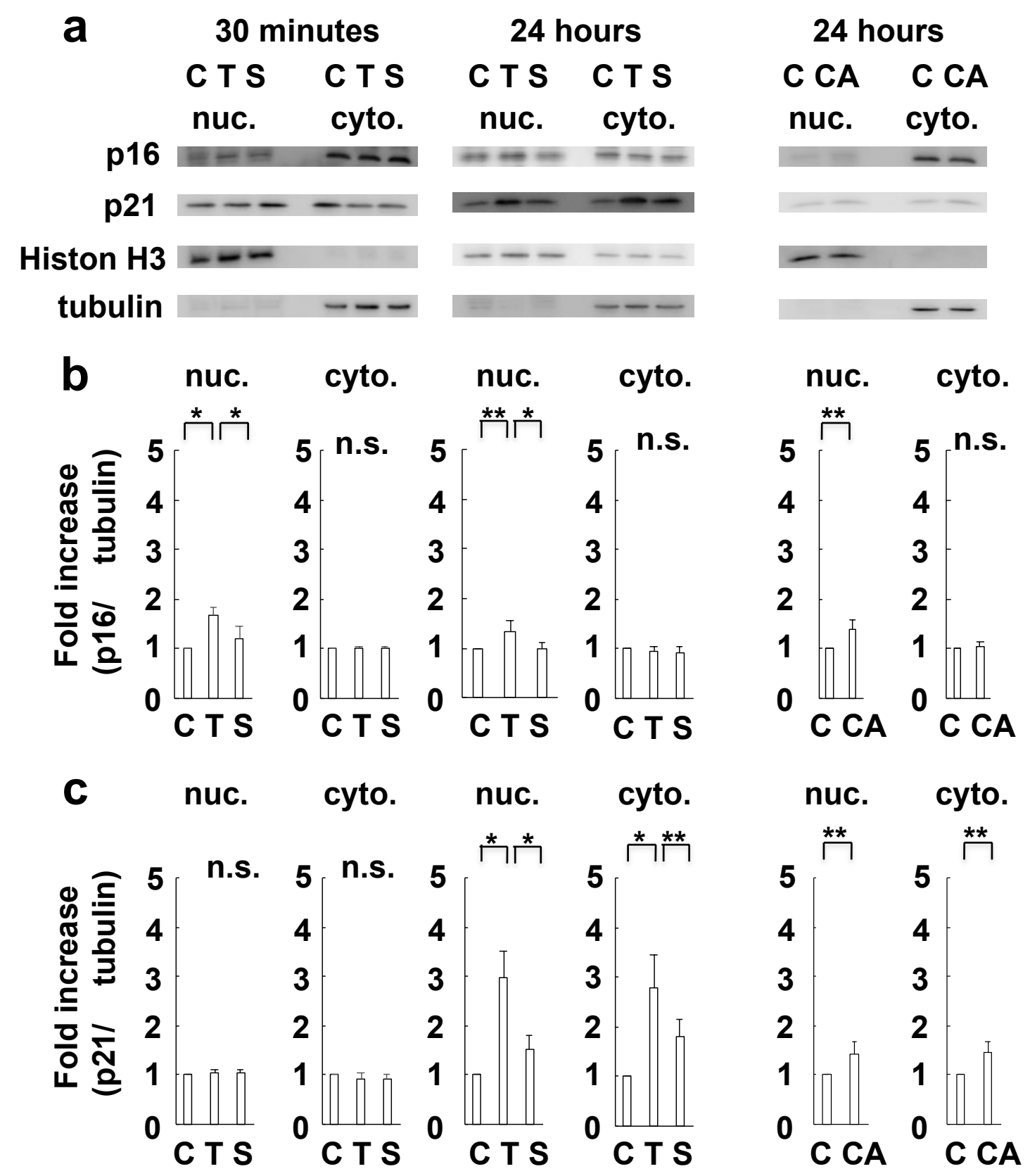

Figure 4. Nuclear translocation of p16 induced by the activation of

TGF- $\beta 1-S m a d 3$ pathway in endothelial cells. 
(a) Representative pictures of western blot analysis of p16 and p21 expression in the nucleus and cytoplasm of endothelial cells induced by the stimulation of TGF- $\beta 1$-Smad3 pathway. (b, c) Activation of TGF- $\beta 1$-Smad3 pathway can translocate p16 to the nuclei in 30 minutes (early phase), while it can increase the expression of p21 in endothelial cells in 24 hours (late phase) $(\mathrm{N}=4)$. C: Control. T: TGF- $\beta 1$. S: SB431542. CA: constitutive active Smad3. nuc.: nucleus. cyto.: cytoplasm. n.s.: not significant. ${ }^{\star} P<0.01$. ${ }^{* *} P<0.05$ (t-test). 


\section{Figure 5}

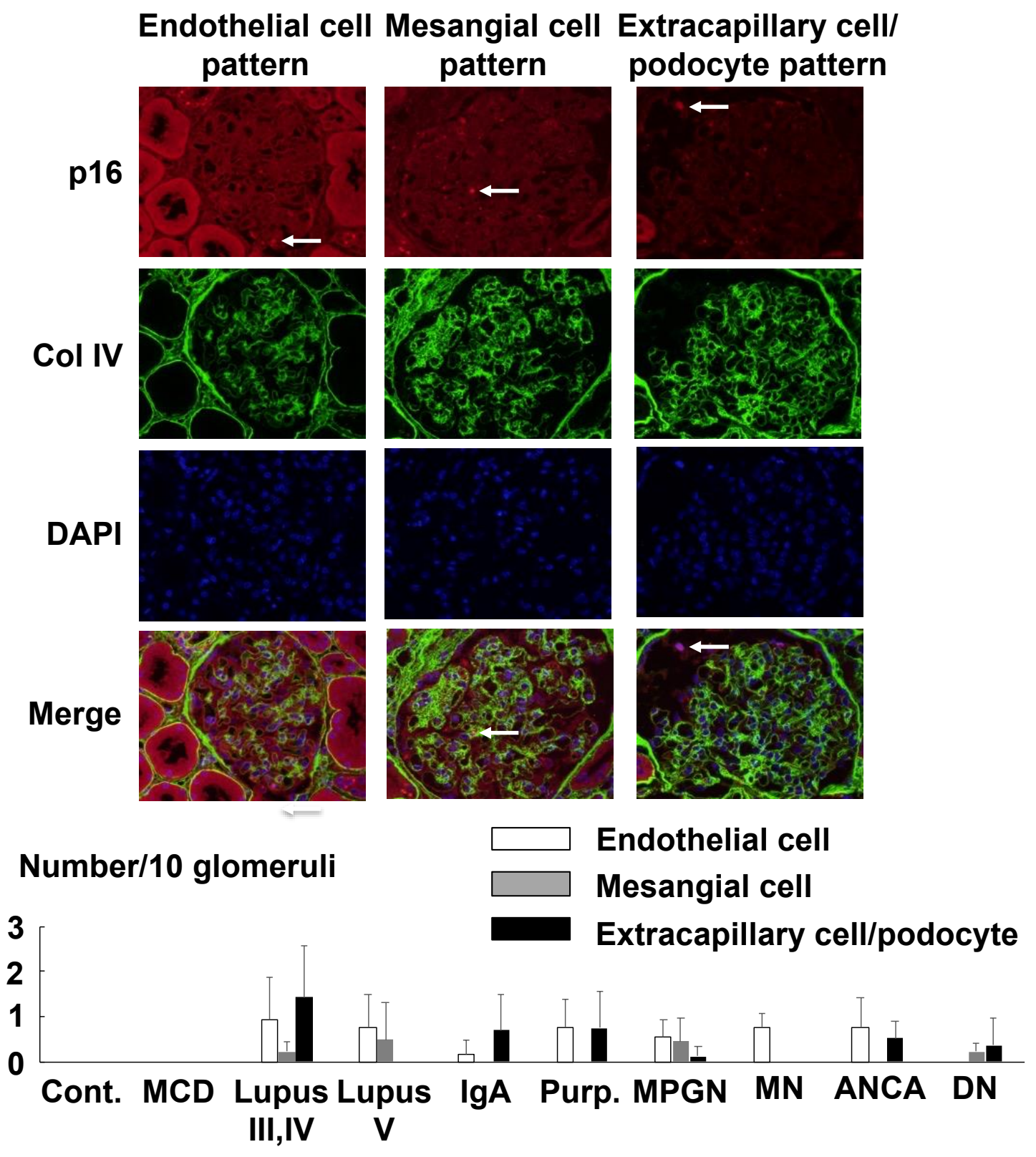

Figure 5. Expression of p16 in patients with kidney disease.

p16 is expressed in endothelial, mesangial cells and extracapillary

cells/podocytes in patients with various kinds of human kidney diseases. 
Representative pictures of each pattern are shown. Col IV: Collagen IV.

Cont.: Control $(N=3)$. MCD: Minimal change disease $(N=3)$.

Lupus III, IV: Systemic lupus nephritis class III or IV $(\mathrm{N}=6)$.

Lupus V: Systemic lupus nephritis class $V(N=3)$. IgA: $\lg A$ nephropathy $(N=6)$.

Purp.: Purpura nephritis $(\mathrm{N}=4)$.

MPGN: Membranoproliferative glomerulonephritis $(\mathrm{N}=3)$.

$M N$ : Membranous nephropathy $(\mathrm{N}=3)$.

ANCA: ANCA glomerulonephritis $(\mathrm{N}=6)$.

DN: Diabetic nephropathy $(\mathrm{N}=6)$.

White column: Endothelial cells. Grey column: Mesangial cells.

Black column: Extracapillary cells and podocytes. 


\begin{tabular}{|c|c|c|c|}
\hline & Number $(\mathrm{N})$ & Females $(\mathrm{N})$ & $\begin{array}{l}\text { Age } \\
\text { (mean } \pm \mathrm{SD}) \text { (years) }\end{array}$ \\
\hline Control & 3 & 2 & $36.0 \pm 24.3$ \\
\hline MCD & 3 & 2 & $52.7 \pm 23.2$ \\
\hline Lupus class III or IV & 6 & 4 & $38.5 \pm 13.5$ \\
\hline Lupus class V & 3 & 3 & $35.7 \pm 7.6$ \\
\hline IgA nephropathy & 6 & 4 & $43.3 \pm 8.9$ \\
\hline Purpura nephritis & 4 & 1 & $62.0 \pm 12.8$ \\
\hline MPGN & 3 & 2 & $65.3 \pm 16.1$ \\
\hline MN & 3 & 1 & $52.3 \pm 13.2$ \\
\hline ANCA glomerulonephritis & 6 & 4 & $78.2 \pm 7.2$ \\
\hline Diabetic nephropathy & 6 & 1 & $62.8 \pm 11.8$ \\
\hline
\end{tabular}

Table 1. The characteristics of the subjects included in this study.

MCD: Minimal change disease. Lupus: Systemic lupus nephritis. MPGN:

Membranoproliferative glomerulonephritis. MN: Membranous nephropathy. 


\section{Figures}

\section{Figure 1}
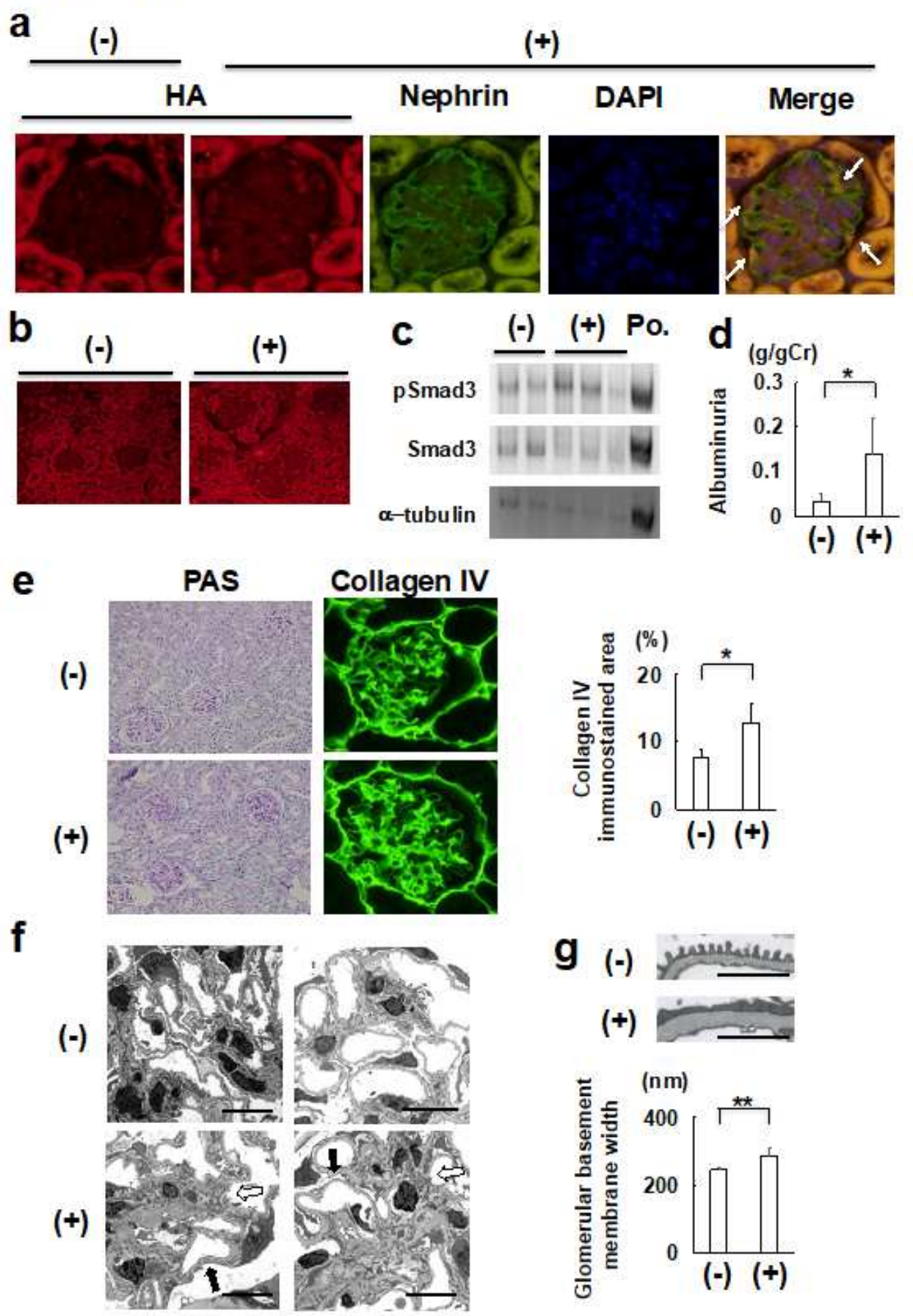

Figure 1

Characterization and pathological changes of podocyte-specific TGF- $\beta 1$ overexpression mice. (a) TGF- $\beta 1$ was expressed in podocytes. HA-tag was conjugated with bioactive porcine TGF- $\beta 1$ in PodCre(+) TGF mice. HA-tag merged with nephrin (podocyte marker). (b) Diffuse expression of HA-tag conjugated TGF- 
$\beta 1$ was observed in the kidney of PodCre(+) TGF mice. (c) Representative pictures of western blot analysis of glomeruli protein. Smad3 was phosphorylated in glomeruli in PodCre(+) TGF mice. (d) Urine albumin excretion was significantly increased in PodCre $(+)$ TGF mice. $(\mathrm{N}=6$ in control mice, $\mathrm{N}=8$ in PodCre(+) TGF mice). *P $<0.01$ (Man-Whitney's U test). (e) Representative pictures of periodic acid-schiff (PAS) stain and collagen IV immunohistochemistry in PodCre(+) TGF mice. PodCre(+) TGF mice showed a significant increase in collagen IV immunostained area $(\mathrm{N}=6$ in control mice, $\mathrm{N}=8$ in PodCre $(+)$ TGF mice). *P $<0.01$ (t-test). (f) Representative pictures of electron microscopy. PodCre(+) TGF mice showed mesangial expansion (white arrow) and diffuse foot process effacement (black arrow). Scale bar: $10 \mu \mathrm{m}$. (g) Representative pictures of glomerular basement membrane by using electron microscopy and quantitative evaluation of glomerular basement membrane width. PodCre(+) TGF mice showed a significant thickening of glomerular basement membrane $(\mathrm{N}=3$ in control mice, $\mathrm{N}=4$ in PodCre $(+)$ TGF mice). Scale bar: $2 \mu \mathrm{m}$. ${ }^{\star *} \mathrm{P}<0.05$ (t-test). (-): Control mice. (+): Podocyte-specific TGF- $\beta 1$ overexpression mice (PodCre(+) TGF mice). Po.: positive control. pSmad3: phosphorylated Smad3. n.s.: not significant. 


\section{Figure 2}

a

(-)

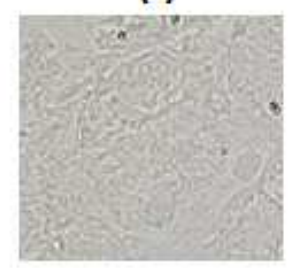

b

$(-)$

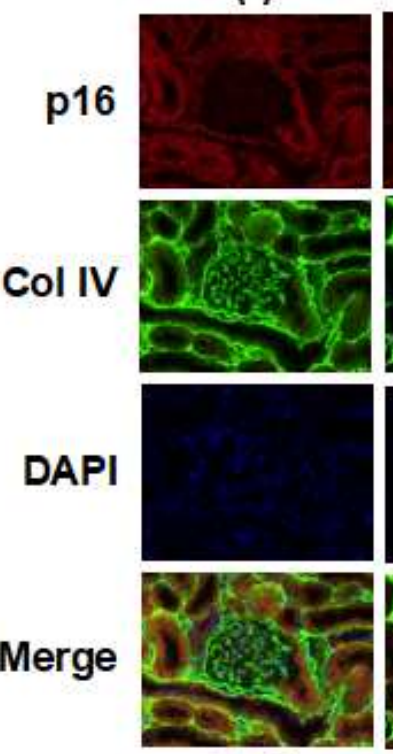

Endo.

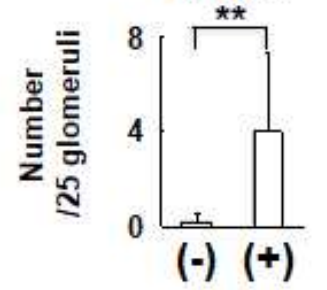

$(-)(+)$

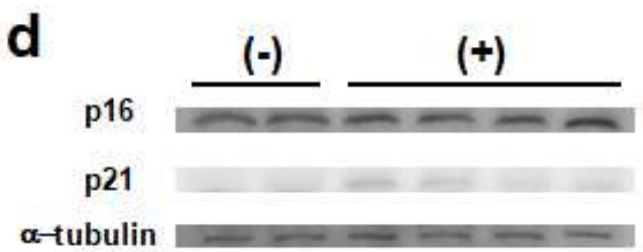

(+)

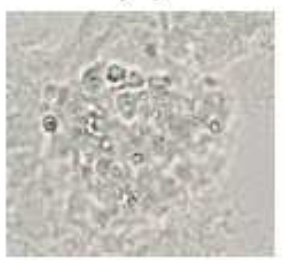

(+)

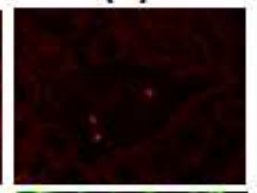

C

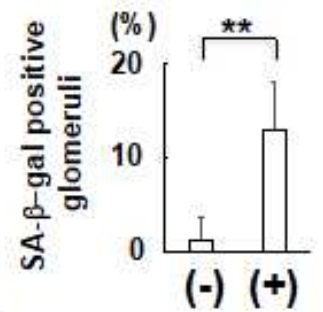

(-) (+)

p21
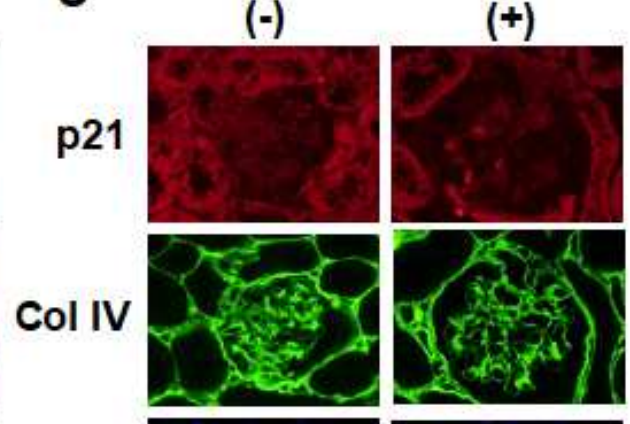

DAPI

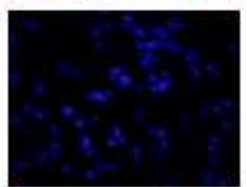

Merge

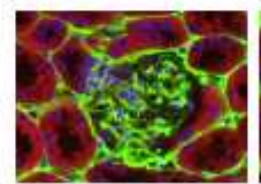

Endo.

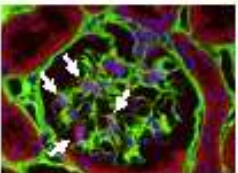

Mes.
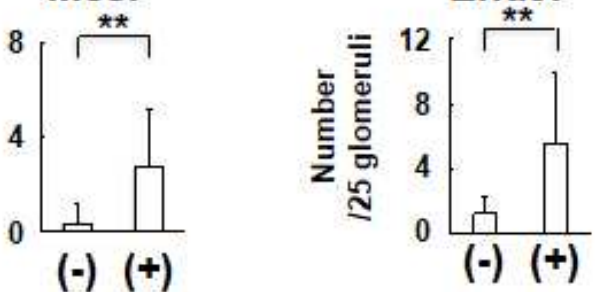

Mes.

12 n.s.
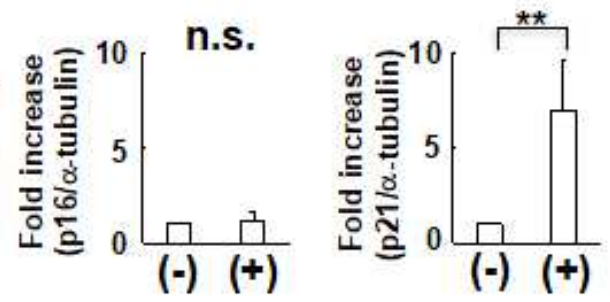

Figure 2

Detection of the markers for cellular senescence in podocyte-specific TGF- $\beta 1$ overexpression mice. (a) Representative pictures of senescence-associated $\beta$-galactosidase staining. Senescence-associated $\beta$ galactosidase activity was significantly increased in PodCre $(+)$ TGF mice. ( $N=3$ in control mice, $N=4$ in PodCre(+) TGF mice). ${ }^{\star} \mathrm{P}<0.05$ (t-test). (b) Representative pictures of p16 immunohistochemistry. PodCre(+) TGF mice had p16 expression mainly in endothelial cells. PodCre(+) TGF mice showed a 
significant increase in p16 immunostained nuclei in endothelial and mesangial cells. ( $N=6$ in control mice, $\mathrm{N}=8$ in PodCre(+) TGF mice). ${ }^{*} \mathrm{P}<0.05$ (t-test). (c) Representative pictures of p21 immunohistochemistry. PodCre(+) TGF mice had p21 expression mainly in endothelial cells. PodCre(+) TGF mice showed a significant increase in p21 immunostained nuclei in endothelial cells. $(N=6$ in control mice, $\mathrm{N}=8$ in PodCre(+) TGF mice). ${ }^{*} \mathrm{P}<0.05$ (t-test). (d) Representative pictures of western blot analysis of p16 and p21 expression in glomeruli. PodCre(+) TGF mice had a significant expression of p21, but not that of p16. ( $N=4$ in control mice, $N=6$ in PodCre(+) TGF mice). ${ }^{\star} P<<0.05$ (t-test). (-):

Control mice. (+): Podocyte-specific TGF- $\beta 1$ overexpression mice (PodCre(+) TGF mice). SA- $\beta$-gal:

Senescence-associated $\beta$-galactosidase. Col IV: Collagen IV. Endo.: Endothelial cell. Mes.: Mesangial cell. n.s.: not significant.

\section{Figure 3}

a

pSmad3

Smad3

p16

p21

\section{$\alpha$-tubulin}

30 minutes
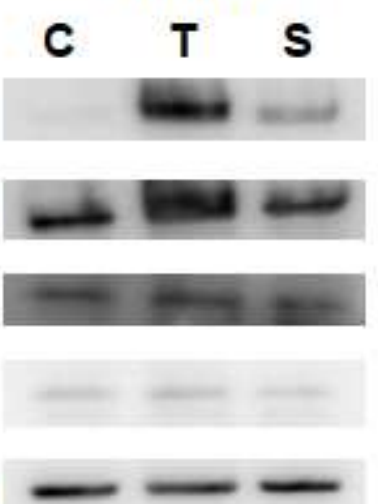

b

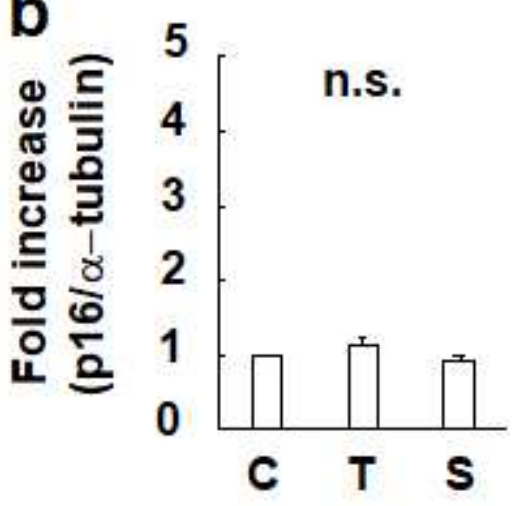

C

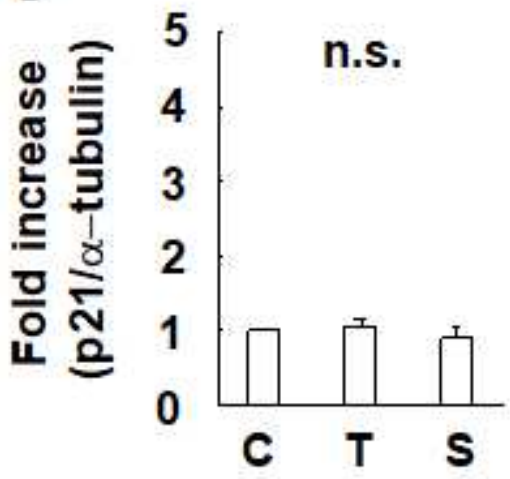

\section{4 hours}

C $\mathrm{T} \mathrm{S}$
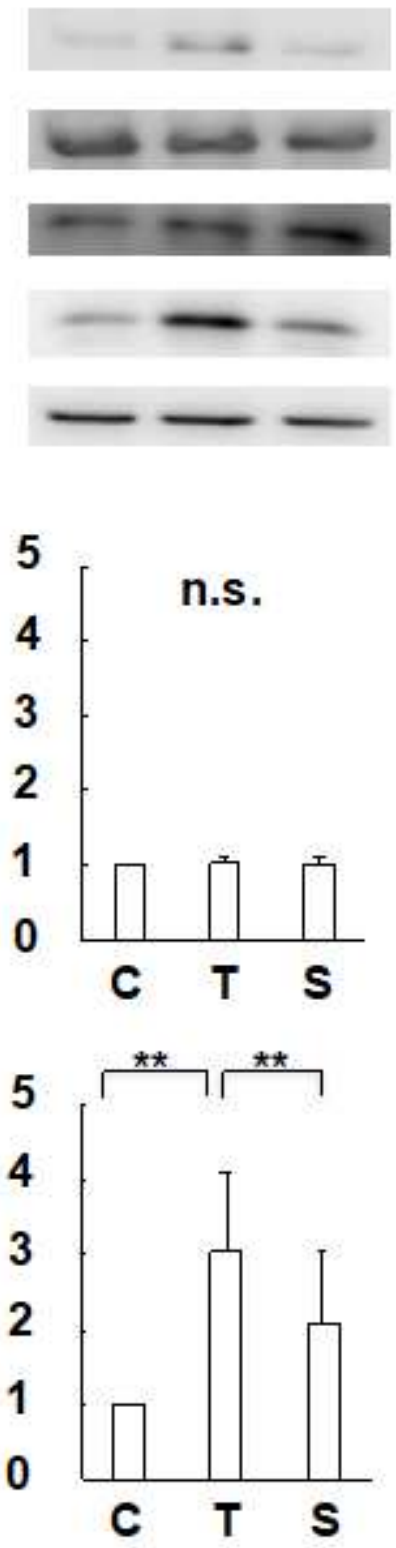

24 hours
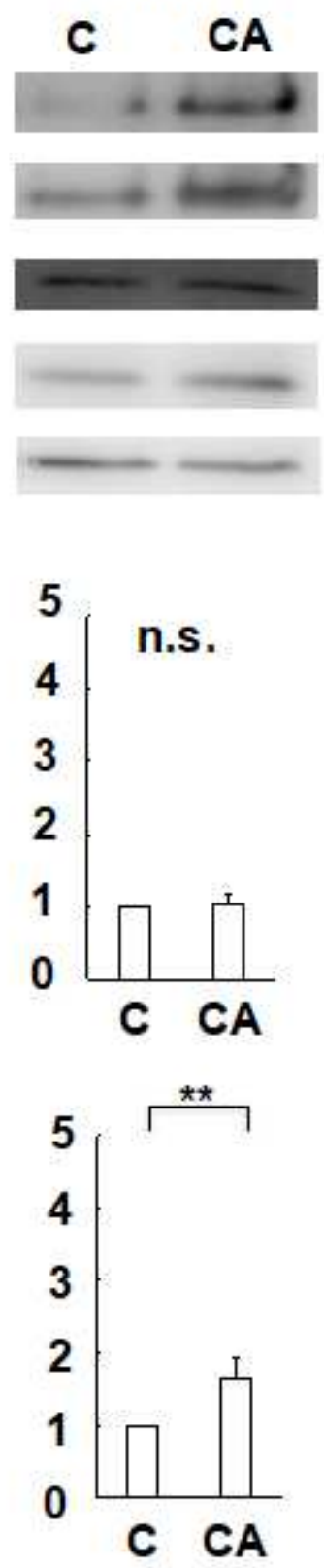
Figure 3

Expression of $\mathrm{p} 16$ and p21 induced by the activation of TGF- $\beta 1$-Smad3 pathway in endothelial cells. (a) Representative pictures of western blot analysis of p16 and p21 expression in endothelial cells induced by the stimulation of TGF- $\beta 1$-Smad3 pathway. (b, c) Activation of TGF- $\beta 1$-Smad3 pathway can increase the expression of p21 in 24 hours (late phase), but not that of p16 in endothelial cells $(N=3)$. C: Control. T: TGF- 31 . S: SB431542. CA: constitutive active Smad3. pSmad3: phosphorylated Smad3. n.s.: not significant. ${ }^{*} \mathrm{P}<0.05$ (t-test).

\section{Figure 4}

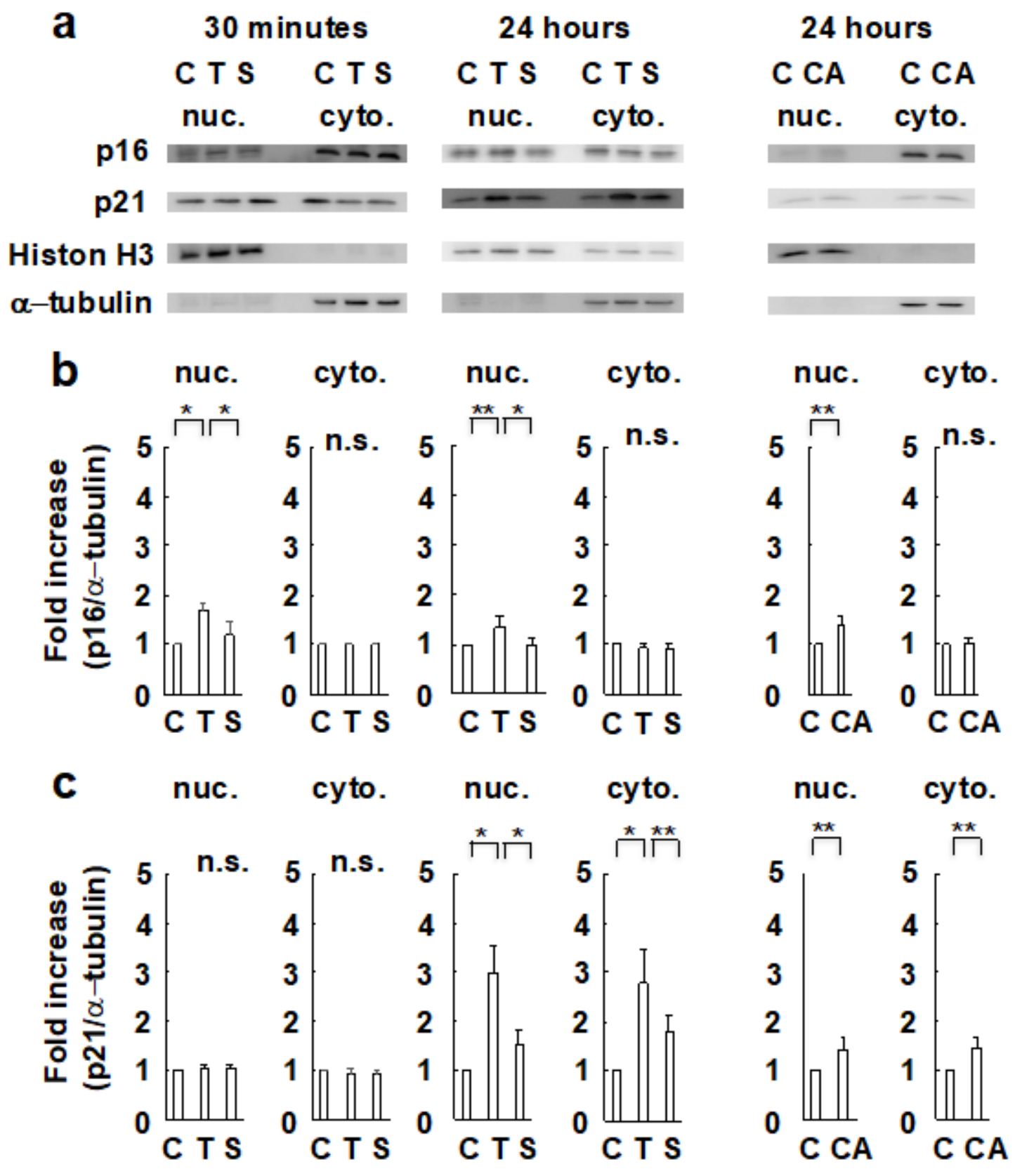

Figure 4 
Nuclear translocation of p16 induced by the activation of TGF- 31 -Smad3 pathway in endothelial cells. (a) Representative pictures of western blot analysis of p16 and p21 expression in the nucleus and cytoplasm of endothelial cells induced by the stimulation of TGF- $\beta 1$-Smad3 pathway. (b, c) Activation of TGF- $\beta 1$ Smad3 pathway can translocate p16 to the nuclei in 30 minutes (early phase), while it can increase the expression of p21 in endothelial cells in 24 hours (late phase) $(\mathrm{N}=4)$. C: Control. T: TGF- $\beta 1$. S:

SB431542. CA: constitutive active Smad3. nuc.: nucleus. cyto.: cytoplasm. n.s.: not significant. ${ }^{*}<0.01$. **P $<0.05$ (t-test).

\section{Figure 5}

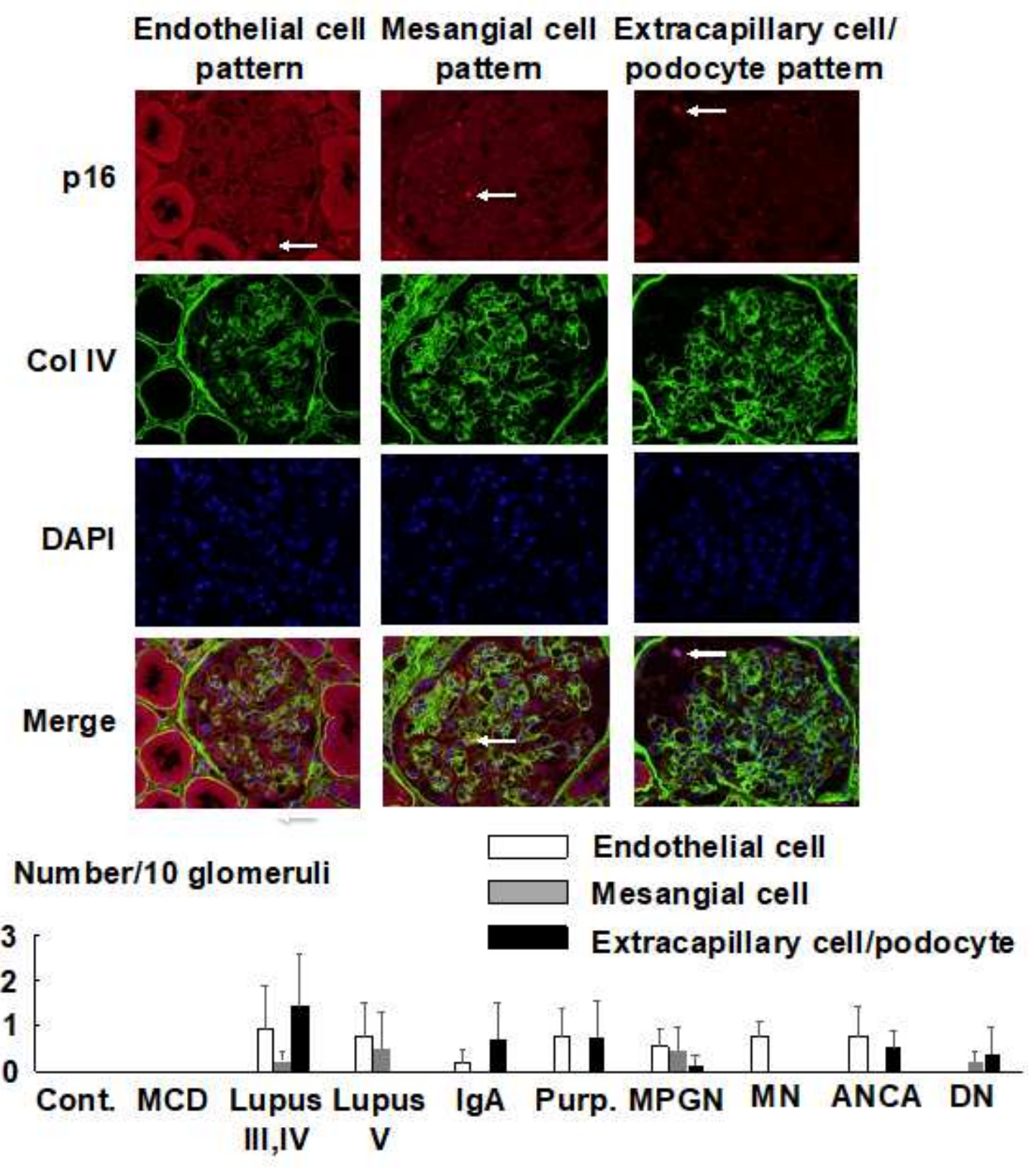

Figure 5 
Expression of p16 in patients with kidney disease. p16 is expressed in endothelial, mesangial cells and extracapillary cells/podocytes in patients with various kinds of human kidney diseases. Representative pictures of each pattern are shown. Col IV: Collagen IV. Cont.: Control $(\mathrm{N}=3)$. MCD: Minimal change disease ( $\mathrm{N}=3)$. Lupus III, IV: Systemic lupus nephritis class III or IV $(\mathrm{N}=6)$. Lupus V: Systemic lupus nephritis class $V(N=3)$. IgA: IgA nephropathy $(N=6)$. Purp.: Purpura nephritis $(N=4)$. MPGN:

Membranoproliferative glomerulonephritis $(N=3)$. MN: Membranous nephropathy $(N=3)$. ANCA: ANCA glomerulonephritis $(N=6)$. DN: Diabetic nephropathy $(N=6)$. White column: Endothelial cells. Grey column: Mesangial cells. Black column: Extracapillary cells and podocytes.

\section{Supplementary Files}

This is a list of supplementary files associated with this preprint. Click to download.

- Supplementarylnformation.pdf 